\title{
Menses Duration
}

National Cancer Institute

\section{Source}

National Cancer Institute. Menses Duration. NCI Thesaurus. Code C156586.

The number of days during which menses is expelled from the uterus. 